\title{
Fatty acid profile, $\alpha$-tocopherol content and total antioxidant activity of oil extracted from Nigella sativa seeds.
}

\begin{abstract}
Nigella sativa (N. sativa) is popularly known as the black seed, a herb that has traditionally been used for centuries in many parts of the world. It has gained popularity due to its potential health benefits. However, more scientific data is needed to support the various health claims. This study was carried out to determine the fatty acid profile, $\alpha$-tocopherol content and to evaluate the antioxidant activity of seed oil samples from three different regions in Yemen namely Marib, Sadah and Taiz. N. sativa seeds oil was extracted using three different solvents (n-hexane, petroleum ether and chloroform: methanol 2:1 v/v) and the fatty acids composition was analyzed using gas chromatography, while the $\alpha$-tocopherol was determined using HPLC. Ferric thiocyanate (FTC) and thiobarbituric acid (TBA) methods were used to evaluate the antioxidant activity of the seeds oil. Results indicated that N. sativa seeds contain high amount of oil (30-48\%) and the major unsaturated fatty acids were linoleic acid (57.96, 58.04 and 57.04\%) followed by oleic acid (21.49, 20.87 and 20.60\%), while the main saturated fatty acids were palmitic $(11.56,11.23$ and $11.22 \%)$, followed by stearic and myristic acids in Marib, Taiz and Sadah samples respectively. Oil extracts exhibited strong antioxidant properties when compared to $\alpha$-tocopherol with $78-82 \%$ inhibition in the FTC method and $70-80 \%$ in the TBA assays. The oil extracts were found to be rich in $\alpha$-tocopherol content $290 \pm 1.5,170 \pm 0.40$ and $120 \pm 0.15 \mathrm{mg} / 100 \mathrm{~g}$, in Marib, Sadah and Taiz samples, respectively. Present results suggest that N. sativa seeds contain high amount of antioxidants that are essential for health and preventing numerous diseases.
\end{abstract}

Keyword: $\alpha$-tocopherol; Fatty acids profile; Nigella sativa; Total antioxidant activity. 